\title{
Large jets from small-scale magnetic fields ${ }^{\star}$
}

\begin{abstract}
R. Moll
Max-Planck-Institut für Astrophysik, Karl-Schwarzschild-Str. 1, 85748 Garching, Germany

e-mail: rmo@mpa-garching.mpg.de

Received 25 August 2009 / Accepted 28 November 2009

ABSTRACT

We consider the conditions under which a rotating magnetic object can produce a magnetically powered outflow in an initially unmagnetized medium stratified under gravity. 3D MHD simulations are presented in which the footpoints of localized, arcade-shaped magnetic fields are placed in rotation. We show how the effectiveness of producing a collimated magnetically powered outflow depends on the rotation rate, and both the strength and the geometry of the field. The flows produced by uniformly rotating, non-axisymmetric fields are found to consist mainly of buoyant plumes heated by the dissipation of rotational energy. Collimated magnetically powered flows are formed if the field and the rotating surface are arranged such that a toroidal magnetic field is produced. This requires that the arcades' footpoints are differentially rotating. These jets are well-collimated and we follow their propagation through the stratified atmosphere over a distance 100 times the source size. The magnetic field is tightly wound and its propagation is dominated by the development of non-axisymmetric instabilities. We observe a Poynting flux conversion efficiency of over $75 \%$ in the longest simulations. Applications to the collapsar model and protostellar jets are discussed.
\end{abstract}

Key words. magnetohydrodynamics (MHD) - ISM: jets and outflows - Herbig-Haro objects - galaxies: jets gamma-ray burst: general

\section{Introduction}

Models and simulations of jets produced by rotating magnetic fields generally assume an ordered, axially symmetric largescale field of uniform polarity anchored in the central engine, based on either the original models of jets from accretion disks by Bisnovatyi-Kogan \& Ruzmaikin (1976) and Blandford (1976), or the magnetic supernova model of LeBlanc \& Wilson (1970). While these ordered fields are the most effective ones for producing jets, the question of whether they actually exist in accretion disks or the core of a star remains open. The largest scale on which magnetorotational (MRI) turbulence in accretion disks shapes the magnetic field structure is determined by the disk thickness, which in turn is much smaller than jets. Collapsar cores are also small compared to the expected GRB jet, and it is not at all clear why the magnetic fields there should be ordered and axisymmetric. Large-scale fields are not easily trapped by an accretion disk (van Ballegooijen 1989). Without a large-scale field protruding from the disk, one may still hope to launch outflows by twisting fields inside the disk or by magnetic loops that extend into the disk corona as e.g. described by the models of Galeev et al. (1979), Tout \& Pringle (1996) and Uzdensky \& Goodman (2008).

The extensive range of length scales involved is a major problem for jet modeling, as numerical simulations covering all scales remain infeasible. Protostellar jets may be several parsecs long, launched by disks with sizes of $\sim 100 \mathrm{AU}$, i.e., about a factor $10^{4}$ smaller (e.g., Shepherd et al. 2001). Assuming that the "launching scale" is much smaller than the disk, perhaps on the order of $\sim 1 \mathrm{AU}$, one discovers an even greater contrast in length scales. Assuming that jets in AGN are launched at a few Schwarzschild radii from the central black hole and taking

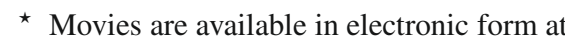
http: //www . aanda . org
Cygnus A as an example, the ratio of the jet length to the size of the engine is about $10^{6}$ (Krichbaum et al. 1998; Tadhunter et al. 2003). The final jet properties are determined before it becomes ballistic, probably on scales that are somewhat smaller than that of the largest visible structures, but still considerably larger than the central engine.

There is numerical evidence that small-scale fields can also be used to generate jets. Most of these simulations are promising in terms of outflow production but limited to the immediate surroundings of the outflow-forming disk. Axisymmetric simulations of outflows generated with small magnetic loops were performed by Romanova et al. (1998), Turner et al. (1999) and Kudoh et al. (2002). In their 3D simulations of accretion flows, Kato et al. (2004) demonstrated that an initially poloidal magnetic field confined within a rotating torus surrounding an accreting black hole can produce a transient outflow driven by accumulated toroidal fields in the form of a "magnetic tower" (Lynden-Bell 2003). De Villiers et al. (2005) showed in simulations that loops of poloidal field in an accreting torus can generate a large-scale poloidal field as the field lines are stretched out in an axial outflow. The inflation and disruption of a magnetic loop outside a disk, caused by the generation of a toroidal field through differential rotation, was observed by Fendt (2009) in simulations of outflows from star-disk magnetospheres. The generation of magnetic flows within unmagnetized surroundings has also been studied in laboratory experiments (Hsu \& Bellan 2005; Ciardi et al. 2009); these jets are strongly affected by current-driven instabilities.

The aim of the calculations presented here is to determine whether small-scale fields in the form of loops anchored in a rotating disk can be used to produce jets of significant length (relative to the size of the source). In the cases studied, the flows propagate and are confined within an external unmagnetized atmosphere (as opposed to Moll et al. 2008 and Moll 2009, 


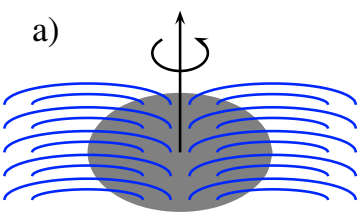

with shear

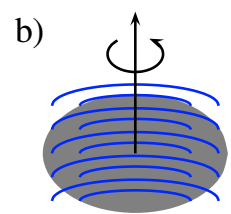

shifted, no shear c)

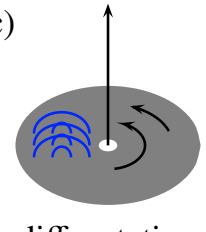

diff. rotation
Fig. 1. Considered magnetic field geometries. In case (a), the field lines are anchored at one end in a rigidly rotating disk. In case (b), the field lines' footpoints are either both anchored in the disk, or they are both anchored outside of it. In case (c), the field emerges from a limited area inside the disk, which is differentially rotating.

hereafter Papers I and II, where we studied jets embedded in a large-scale magnetically dominated environment). While still idealized conditions, they are appropriate for environments such as protostellar jets launched into a dense cloud or GRB jets launched by a collapsar core. We investigate here the circumstances in which jetlike flows are formed that penetrate the atmosphere instead of dissipating in it. As this is also a question of (non-axisymmetric) stability, three-dimensional simulations are necessary. One of the questions to be answered is whether models of jets from small-scale magnetic fields are a viable alternative to those based on the twisting of large-scale fields.

\section{Models}

The primary ingredients in all of our models are a rotating disk, implemented as a boundary condition, and magnetic field loops anchored in and sticking out of the disk. The magnetic field geometries considered are sketched in Fig. 1. In case (a), some of the loops have one footpoint inside the disk while the other is anchored outside. The resulting shear motion of the footpoints generates a toroidal magnetic field. In case (b), the loops are arranged so that they are not sheared. Here, a toroidal field can only be produced by the inertia of the material above the disk, as in conventional models of jets from large-scale magnetic fields. This case would represent a model for a (solidly) rotating stellar core inside a nonmagnetic envelope. Finally, we consider a case in which all loops are anchored in the disk and shear is created by differential rotation (case c). This case is more representative of small-scale fields generated in an accretion disk.

The magnetic field loops are established by either a suitable potential field, imposed as an initial condition (setup D), or continuous "injection" of the field from below the disk (setup E, see Fig. 3 and Sect. 3.1.2 for details). While the magnetic field geometry is similar in both cases, setup E (for "emerging field") represents an idealization of the field loops emerging from magnetic turbulence in an accretion disk.

The model chosen to be the initial condition of the atmosphere is a hydrostatic equilibrium stratification in the gravitational potential of a point mass (the origin). Temperature $T$ and density $\rho$ vary with distance $r$ from the point mass as $r^{-1}$ and $r^{-3}$, respectively. The temperature is thus a constant fraction of the virial temperature, as in advection-dominated accretion flows. The density profile has the property that the amount of mass in a cone centered on the origin is constant per decade in distance $r$. The stratification is thus scale-free, and a prospective jet encounters the same amount of atmosphere mass per decade traveled. For the equation of state used in the calculations, i.e., of an ideal gas with ratio of specific heats $\gamma=5 / 3$, the stratification is convectively stable.

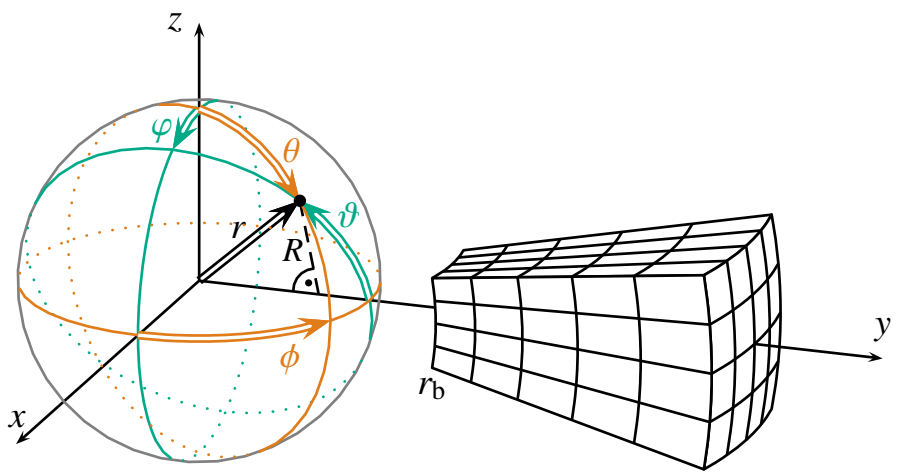

Fig. 2. Sketch of the coordinate system and the computational grid. The jets propagate in the equatorial direction of the grid coordinates $(r, \theta, \phi)$. To describe the results, an alternative spherical coordinate system $(r, \vartheta, \varphi)$ is used, which assumes the jet's central axis to be the polar axis.

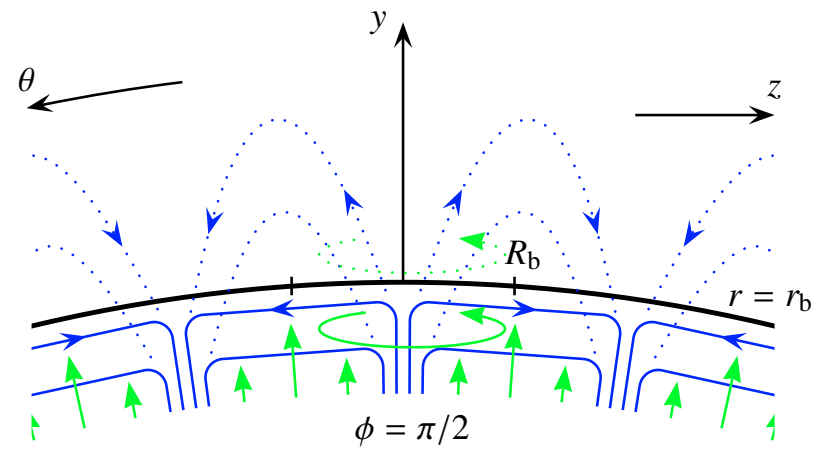

Fig. 3. Schematic of simulation setup E described in Sect. 3.1.2, in the variant sketched in Fig. 1a. A vertical velocity field (green arrows) pushes the magnetic field (blue lines) from the lower boundary $\left(r=r_{\mathrm{b}}\right)$ into the computational volume. An azimuthal velocity field within $R \leq R_{\mathrm{b}}$ twists the created magnetic arcades (dotted blue lines), which are anchored at one end in the rotating region.

\section{Methods}

\subsection{Numerical MHD solver, grid, and coordinates}

We numerically solved the ideal adiabatic MHD equations with a static external gravitational potential $\Phi \propto r^{-1}$ on a spherical grid $(r, \theta, \phi)$. The jets propagate in the equatorial direction along the $y$-axis $(\theta=\phi=\pi / 2)$, about which the computational volume covers a range $\Delta \theta=\Delta \phi$ in the angular directions (see Fig. 2). The spacing is uniform in $\theta$ and $\phi$ and logarithmic in $r$. This grid is more economical than a Cartesian one because it expands in the jet direction. Unlike a spherical grid in polar direction $z$, it is free of singularities that may cause numerical problems or artifacts.

Since jet physics is more conveniently described in a coordinate system which takes the jet's axis $y$ as the polar axis, we introduce another spherical coordinate system $(r, \vartheta, \varphi)$ for that purpose, see also Fig. 2. $R:=r \sin \vartheta$ denotes the orthogonal distance to that axis (cylindrical radius).

The simulations were performed with a newly developed Eulerian MHD code (Obergaulinger 2008). It is based on a flux-conservative finite-volume formulation of the MHD equations and the constraint transport scheme that maintains a divergence-free magnetic field (Evans \& Hawley 1988). Using high-resolution shock capturing methods (e.g., LeVeque 1992), it allows a choice of various optional high-order reconstruction algorithms and approximate Riemann solvers based on the 
multi-stage method (Toro \& Titarev 2006). The simulations presented here were performed using a fifth order monotonicitypreserving reconstruction scheme (Suresh \& Huynh 1997), the HLL Riemann solver (Harten 1983), and third order Runge-Kutta time stepping.

As in other astrophysical MHD simulations, the implicit assumption was made that the magnetic reconnection in the settings studied is of the "fast" type, for which the reconnection implicit in the numerical diffusion of the code may be a fair representation of the subgrid dynamics.

\subsubsection{Setup with an arcade-shaped, exponentially decreasing initial field (D)}

The initial condition of the magnetic field in this case is represented by a two-dimensional potential field that is independent of the $x$-coordinate (see Figs. 2, 3 for the coordinate system) and declines exponentially with height $y$. It is generated by the vector potential

$\boldsymbol{A}=B_{\mathrm{b}} \lambda \sin \left(\frac{z}{\lambda}\right) \exp \left(\frac{r_{\mathrm{b}}-y}{\lambda}\right) \hat{\boldsymbol{e}}_{x}$,

where $\lambda \pi$ is the width of the arcades, $\lambda$ is the scale height of $B=$ $|\boldsymbol{B}|$, and $B_{\mathrm{b}}$ is the field strength at the intersection of the lower boundary $r=r_{\mathrm{b}}$ with the central axis $(y)$. To avoid numerical problems at the lateral boundaries, we limit the number of arcs to 4 , setting $\boldsymbol{A}=\boldsymbol{B}=0$ when $|z|>2 \lambda \pi$ or $|x|>2 \lambda \pi$.

The magnetic field is embedded in a spherically stratified atmosphere with $p \propto r^{-4}$ and $\rho \propto r^{-3}$, held in hydrostatic equilibrium by the static gravitational field $\Phi \propto r^{-1}$ of a point mass at the coordinate origin. Temperature and sound speed vary as $T \propto r^{-1}$ and $c_{\mathrm{s}} \propto r^{-1 / 2}$, respectively. In the outer, unmagnetized region $(|x|,|z|>2 \lambda \pi)$, we compensated for the absence of magnetic pressure by increasing the gas pressure. To maintain hydrostatic equilibrium, the density was also increased correspondingly.

At the lower $\left(r=r_{\mathrm{b}}\right)$ boundary, we maintain, by means of "ghost cells" outside the computational domain, an azimuthal velocity field $\boldsymbol{v}=v_{\varphi} \hat{\boldsymbol{e}}_{\varphi}$ corresponding to rigid rotation: $v_{\varphi}=$ $v_{\varphi, \mathrm{b}}^{\max } R / R_{\mathrm{b}}$ for $R \leq R_{\mathrm{b}}$ and 0 elsewhere. All quantities except for $\boldsymbol{B}$ are fixed at their initial values in the ghost cells; $\boldsymbol{B}$ is extrapolated from the interior of the domain. At the sides $(\theta$ and $\phi)$ and top (upper $r$ ) of the domain, we use open boundary conditions that allow an almost force-free outflow of material and cause no evident artifacts in the form of reflections.

We select $\lambda$ such that in the $x=0$ plane, $B_{y}$ is positive for $|z|<R_{\mathrm{b}}$ and negative for $R_{\mathrm{b}}<|z|<2 R_{\mathrm{b}}$, i.e., arcades that begin at $|z|<R_{\mathrm{b}}$ from the $r=r_{\mathrm{b}}$ surface have their second footpoint outside the rotating region. This is achieved by choosing the length scale of the arcade to be $\lambda=2 R_{\mathrm{b}} / \pi$.

\subsubsection{Setup with magnetic field arcades emerging from the bottom boundary (E)}

In this case, the initial condition is again an equilibrium stratification with $p \propto r^{-4}, \rho \propto r^{-3}$, and $\Phi \propto r^{-1}$. However, unlike in setup $\mathrm{D}$, the atmosphere is completely unmagnetized.

The magnetic field enters the domain through the lower boundary $r=r_{\mathrm{b}}$, the conditions of which are determined by ghost cells at $r<r_{\mathrm{b}}$. There, we impose a transverse magnetic field $B_{\theta}\left(\approx B_{z}\right)$ of constant amplitude $B_{\mathrm{b}}$ and a polarity that alternates with $z=r_{\mathrm{b}} \cos \theta$ in step sizes of $2 R_{\mathrm{b}}$, which is the diameter of the rotating surface (described below). Where the polarity of
Table 1. Normalization units.

\begin{tabular}{ccc}
\hline \hline Quantity & Symbol(s) & Unit \\
\hline length & $x, y, z, r, R$ & $l_{0}$ \\
gas pressure & $p$ & $p_{0}$ \\
density & $\rho$ & $\rho_{0}$ \\
velocity & $v$ & $c_{\mathrm{s} 0}=\sqrt{\gamma p_{0} / \rho_{0}}$ \\
time & $t$ & $t_{0}=l_{0} / c_{\mathrm{s} 0}$ \\
energy flow rate & $\mathcal{E}$ & $p_{0} l_{0}^{3} / t_{0}$ \\
magnetic flux density & $B$ & $B_{0}=\sqrt{8 \pi p_{0}}$ \\
\hline
\end{tabular}

$B_{\theta}$ changes, the solenoidality of the magnetic field is maintained by an appropriate $B_{r}$. The field lines thus have the shape sketched in Fig. 3. An approximate equilibrium is maintained by lowering the gas pressure by the value of the magnetic pressure, as far as this is possible ( $p$ must not be negative). For this to be achieved, $\beta_{\mathrm{b}}:=8 \pi p_{\mathrm{b}} / B_{\mathrm{b}}^{2}$ must be greater than one, which limits the possible field strengths to $B_{\mathrm{b}}<1$ in our system of units (described below).

To model the emergence of magnetic fields into the atmosphere, we impose the radial velocity field

$v_{r}=0.9 c_{\mathrm{s}, \mathrm{b}}\left|\sin \frac{z \pi}{2 R_{\mathrm{b}}}\right|$

in the ghost cells of the lower boundary. The injection velocity is too small to form a jet by itself: the maximum amounts to $41 \%$ of the escape velocity at the boundary, gas with this speed gets theoretically as far as $r \approx 1.2 r_{\mathrm{b}}$ without acceleration other than gravity. In addition to the radial velocity field, we maintain an azimuthal velocity field $v_{\varphi} \propto R$ (rigid rotation) within $R \leq R_{\mathrm{b}}$. The shape of the emerging magnetic field is sketched in Fig. 1a. For the other boundaries, we use the same outflow conditions as in the model described in the preceding section.

Two variations in the setup described above have also been studied, corresponding to the cases (b) and (c) described in Sect. 2. In the first, the positions where $B_{\theta}$ changes its polarity, and with it the radial velocity field that injects the magnetic field, are shifted by $R_{\mathrm{b}}$ in the $-z$ direction. Thus, both footpoints of an individual field loop rotate with the same angular velocity. In the second case (c), the magnetic field and vertical velocity in the lower boundary are changed such that only a small, confined arcade in $0.2<R / R_{\mathrm{b}}<0.8$ with a width of $0.2 R_{\mathrm{b}}$ emerges, and the azimuthal velocity field has a Keplerian profile with $v_{\varphi}=v_{\varphi, \mathrm{b}}^{\max } \sqrt{0.1 R_{\mathrm{b}} / R}$ for $0.1 \leq R / R_{\mathrm{b}} \leq 1$. The inner edge $\left(R=0.2 R_{\mathrm{b}}\right)$ of the arcade rotates twice as rapidly as the outer edge $\left(R=0.8 R_{\mathrm{b}}\right)$, the difference in rotation velocity being $v_{\varphi, \mathrm{b}}^{\max } / \sqrt{8}$.

\subsection{Parameters and units}

The models described above contain 6 parameters, $r_{\mathrm{b}}, R_{\mathrm{b}}, \rho_{\mathrm{b}}$, $p_{\mathrm{b}}, B_{\mathrm{b}}$, and $v_{\varphi, \mathrm{b}}^{\max }$, not all of which are independent. We eliminate the dependences by using $l_{0} \equiv 2 R_{\mathrm{b}}, \rho_{0} \equiv \rho_{\mathrm{b}}$ and $p_{0} \equiv p_{\mathrm{b}}$ as units of length, density and pressure, and expressing all physical quantities in terms of these (see Table 1). The remaining 3 parameters can then be expressed as dimensionless numbers. The first of these is $l_{0} / r_{\mathrm{b}}$, which measures the curvature of the rotating surface. In all simulations presented here, this curvature is small and probably does not influence the results significantly. The parameter has technical significance, however, because it also controls the opening of the lateral boundaries. The remaining two numbers are chosen to be $\beta_{\mathrm{b}}:=8 \pi p_{0} / B_{\mathrm{b}}^{2}$, which controls the strength of the magnetic field, and the Mach number 
$\mathrm{M}_{\mathrm{b}}:=v_{\varphi, \mathrm{b}}^{\max } / c_{\mathrm{s} 0}$, which determines the speed of rotation. Note that in the emerging field model $(\mathrm{E}), \beta_{\mathrm{b}}$ is a measure of the field strength but not a local plasma-beta value, since the pressure entering its definition is not measured at the same location as the field strength. For the sake of clarity, we usually omit the units in the presentation of the results. The quantity concerned is then measured in terms of the associated normalization unit listed in Table 1.

\section{Results}

As explained in Sect. 2 above, the calculations were performed using different models of the rotating magnetic field configuration. We find that some of these cases produce long-lived jets, while others produce only transient flows or flows that dissipate in the atmosphere close to the source. The results presented in the following subsection were obtained with setup D (Sect. 3.1.1), and those in the subsequent subsections were obtained with setup E (Sect. 3.1.2).

\subsection{Transient jets with arcade-shaped initial field}

Simulation setup D with the field geometry in Fig. 1a was found to produce extended, collimated outflows. However, the magnetic field at the base of the jet decays in this case (hence the "D") and is not replenished, for which reason these jets are not permanent.

The forced rotation at the lower boundary stretches the magnetic field lines in the azimuthal $(\varphi)$ direction around the axis of rotation $(y)$. The gas is accelerated upwards and the magnetic field assumes a tangled helical structure. A good momentary acceleration was obtained with the parameters $\left(\beta_{\mathrm{b}}=1 / 4, \mathrm{M}_{\mathrm{b}}=\right.$ 18). The magnetic field in this case is depicted in Fig. 4. The jet diameter is resolved with about 35 grid cells in this simulation. The jet attains a height of about 30 times its initial diameter, the factor of expansion being about 7. It reaches velocities that are close to $M_{b}$ and about a factor of 10 above the escape speed. The field lines close at the lower boundary: inside the jet, the radial magnetic field has the same polarity as on the disk, whereas the net radial magnetic flux $\int B_{r} \mathrm{~d} A$, integrated over the $r=$ constant surface, is virtually zero at all times and all radii.

The "absolute flux" $\int\left|B_{r}\right| \mathrm{d} A$ decreases linearly with time close to the lower boundary. Higher temperatures close to the outside of the rotating surface indicate that a substantial amount of the magnetic field is being dissipated there (see Fig. 5). Because of the rotation, the magnetic field assumes a vortexlike structure, at the border of which magnetic field lines of opposite polarities become entangled. This leads to a continuous decay of the magnetic field. This decay of the magnetic field by the wrapping-up of field lines followed by their cancellation by diffusion is called "convective expulsion" in overturning flows (e.g., Zel'dovich 1956; Parker 1963). The magnetic field in the physical source of a successful jet must evidently be of a different nature. This calls for a modification of the boundary conditions at the base of our simulations. These cases are discussed in the following sections.

\subsection{Jets from emerging fields: parameter study}

Simulation setup E, in which the magnetic field loops above the rotating surface are continuously replenished, produced longlasting jets that propagate to considerable distances. Before presenting intensive simulations of long jets in the next two

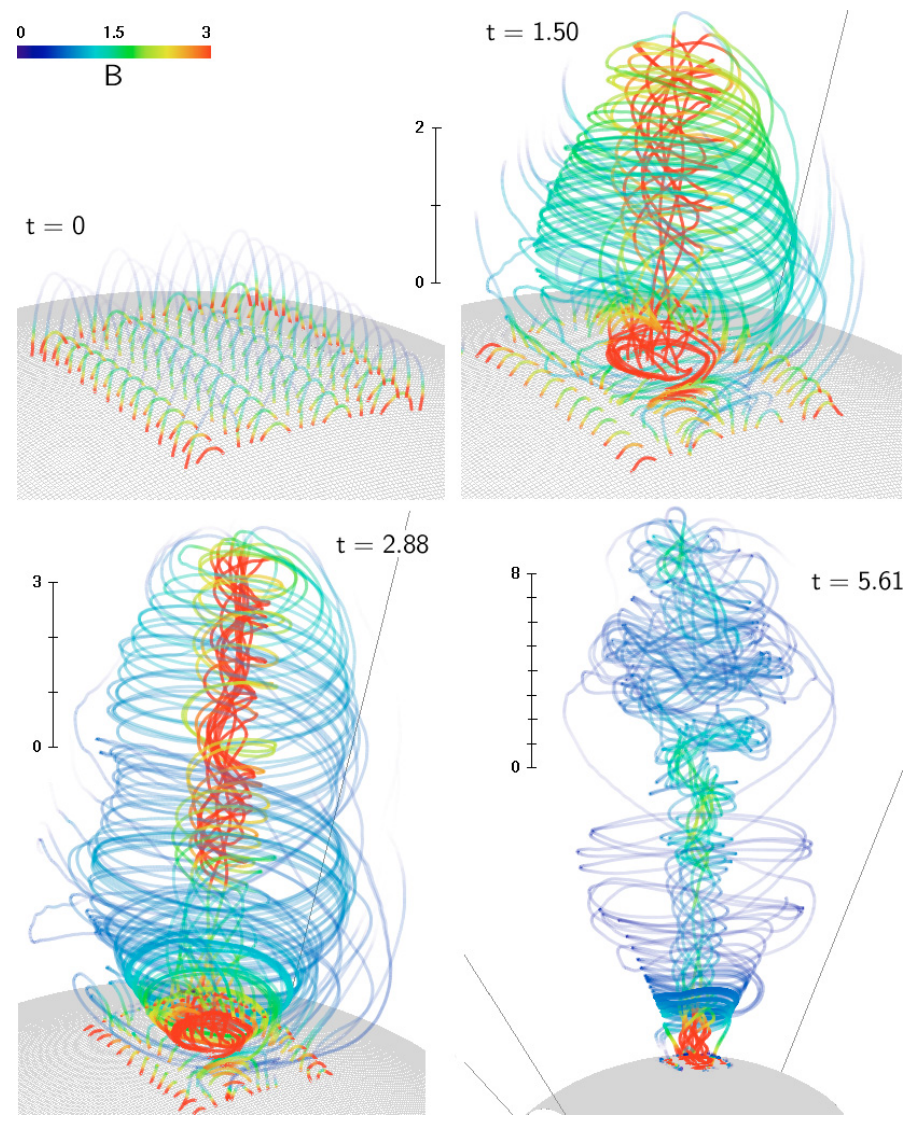

Fig. 4. Selected magnetic field lines in a simulation with an arcadeshaped initial field (setup D). The lines close at the lower boundary, ascending inside the jet and descending outside.

sections, we discuss the influence of the parameters $\left(\beta_{\mathrm{b}}, \mathrm{M}_{\mathrm{b}}\right)$ on the results by comparing a series of smaller, computationally less intensive simulations in which these parameters are varied. Although the proximity of the upper boundary possibly influences the results (see discussion of instabilities below), the smaller simulations provide clear indications of which parameters yield efficient jets.

The simulations cover the relatively short distance of 20 length units (40 disk radii), with $10<r<30$ and $\theta, \phi=$ $\pi / 2 \pm \pi / 9$, the resolution being $256 \times 96 \times 96$. The atmospheric density differs by a factor of 27 between the lower and upper boundaries. The simulations continued until $t \approx 100$. When a jet was successfully launched, this corresponds to about 2-3 passages through the computational volume. The computational cost of these simulations was 1-3 wallclock days on 36 processors with MPI parallelization.

To test the flow's ability to penetrate the atmosphere in the setup with shear, Fig. 1a, we compare the jet velocities halfway through the simulated distance with the escape velocity in Fig. $6^{1}$. The jet velocity decreases with both decreasing rotation velocity $\mathrm{M}_{\mathrm{b}}$ and decreasing magnetic field strength $B_{\mathrm{b}}$ (or increasing $\beta_{\mathrm{b}}$ ). It is, however, far more sensitive to the former parameter: $\beta_{\mathrm{b}}$ must be changed by orders of magnitude before having a significant impact on jet velocity whereas with $M_{b}$, a factor of order unity suffices. The bottom panel in Fig. 6 indicates the flows that pass this test, those exceeding the escape

\footnotetext{
1 Animated plots can be found in the electronic edition of the journal and at http://www.mpa-garching.mpg.de/ rmo/pap3/ index. html
} 

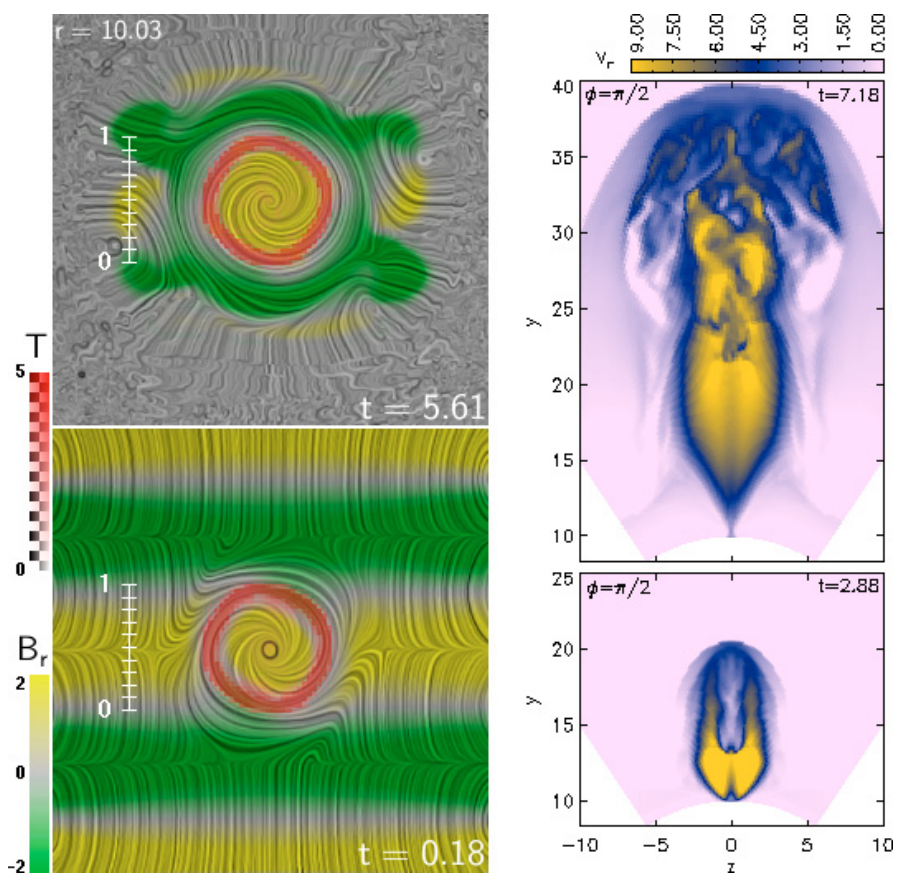

Fig. 5. Left: magnetic field and temperature in a horizontal slice next to the bottom boundary in a simulation with an arcade-shaped initial field (setup D) with shear (Fig. 1a) after 1 (bottom left panel) and 32 revolutions (top left panel). The texture shows the geometry of the parallel field component and the green/yellow coloring indicates the magnitude of the normal component. Regions of higher temperature are tinted in red (lower temperatures are transparent). Right: evolution of the radial velocity in a meridional slice. The jet is eventually "choked off" at the bottom.

speed are indicated in green, the others in red. Failed jets do not reach the upper boundary. The background atmosphere, whose equilibrium is perturbed, tends to fall down on top of them. The fixed conditions at the lower boundary avert a pile-up of thermal energy as downward flowing hot gas vanishes across the boundary and the injected gas has a constant temperature. A delayed onset of a flow because of accumulated heat is unlikely for this reason.

Instabilities occur in all cases where a jet develops. The instabilities develop mainly after the first passage through the computational domain, in the form of helical displacements and/or a change of direction of the whole jet by up to several degrees. The latter are probably modes with wavelengths longer than the computational domain, i.e., one sees only the lower part of what would be a kink if the radial extent of the domain was larger. Jets created by stronger magnetic fields $\left(\beta_{\mathrm{b}} \leq 4.9\right)$ tend to show this kind of incipient instability. Jets from weaker fields move slower and develop pronounced helical deformations within the computational domain. The proximity of the upper boundary does not allow us to draw more conclusive statements about differences in instability behavior within the limits of this parameter study. A series of large, more intensive simulations of the kind presented in the next section would be needed for that.

If the configuration is shifted such that no shear in the magnetic field occurs (Fig. 1b), a jet does not develop. The middle panel in Fig. 7 illustrates this case. The magnetic field lines are unconnected to the surrounding atmosphere and the field is not amplified by shear. This appears to be sufficient to allow them to rotate without producing a magnetically powered flow. However, for very large values of $\mathrm{M}_{\mathrm{b}}$ and $\beta_{\mathrm{b}}$, an outflow forms at the edge of the rotating disk (see bottom panel in Fig. 7). This outflow
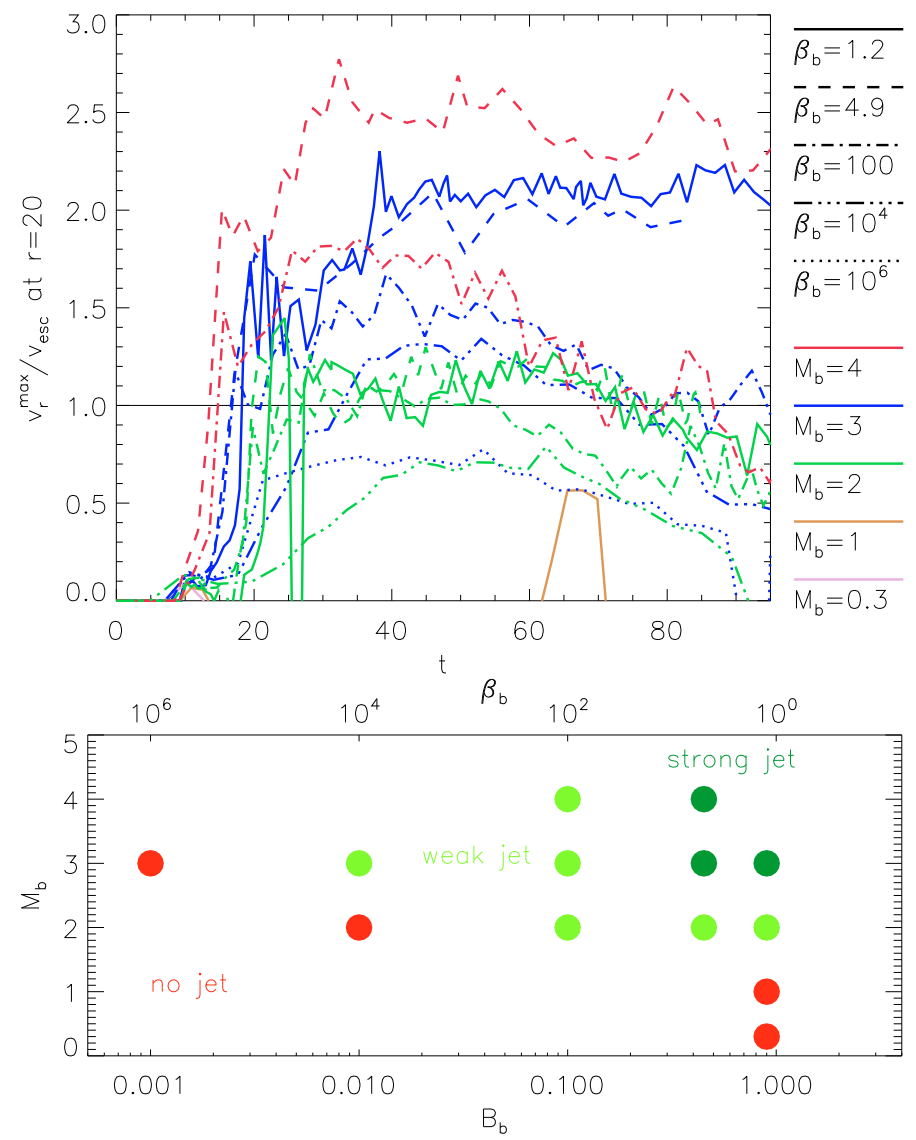

Fig. 6. Top panel: maximum velocity over escape speed for different parameters $\left(\beta_{\mathrm{b}}, \mathrm{M}_{\mathrm{b}}\right)$ in otherwise similar simulations of the emerging field model (E), variant (a). The strength of the jet decreases with a decrease of the field strength (increasing $\beta_{\mathrm{b}}$, represented by different line styles) and with a decrease of the rotation velocity $\left(\mathrm{M}_{\mathrm{b}}\right.$, represented by different colors). Bottom panel: strength of the jets as a function of the rotation rate and field strength parameters.

appears to be driven by thermal buoyancy associated with the dissipation of rotational energy at the disk's edge. Having the shape of a hollow cone with a large opening angle, it can be clearly distinguished from the jets discussed above and in the remainder of this paper.

\subsection{Jets from emerging fields: a large simulation}

The long-range behavior of jets from emerging fields (setup E) in the case of shear (Fig. 1a) was investigated in a large simulation covering the radial range $10<r<110$. Along this range, the atmospheric density decreases by a factor 1300 . The parameters used were $\left(\beta_{\mathrm{b}}=4, \mathrm{M}_{\mathrm{b}}=3\right)$. The jet crosses the upper boundary at the physical time $t \approx 110$, which corresponds to 105 complete rotations of the disk; the simulation was stopped at $t=539$. For this, 19 wallclock days on 64 processors with MPI parallelization were needed, the resolution being $400 \times 160 \times 160$. The lateral boundaries are at $\theta, \phi=\pi / 2 \pm \pi / 6$; the jet remains within these boundaries at all times.

The wound-up magnetic field rises upwards as shown in Fig. 8, a collimated outflow forming in the vertical direction. Not counting the changes in direction caused by instabilities, the jet's half opening angle is about $5^{\circ}$. Inside the jet, the field lines spiral upwards, the radial magnetic flux having the same sign as the radial field on the rotating surface. The total flux is, in 


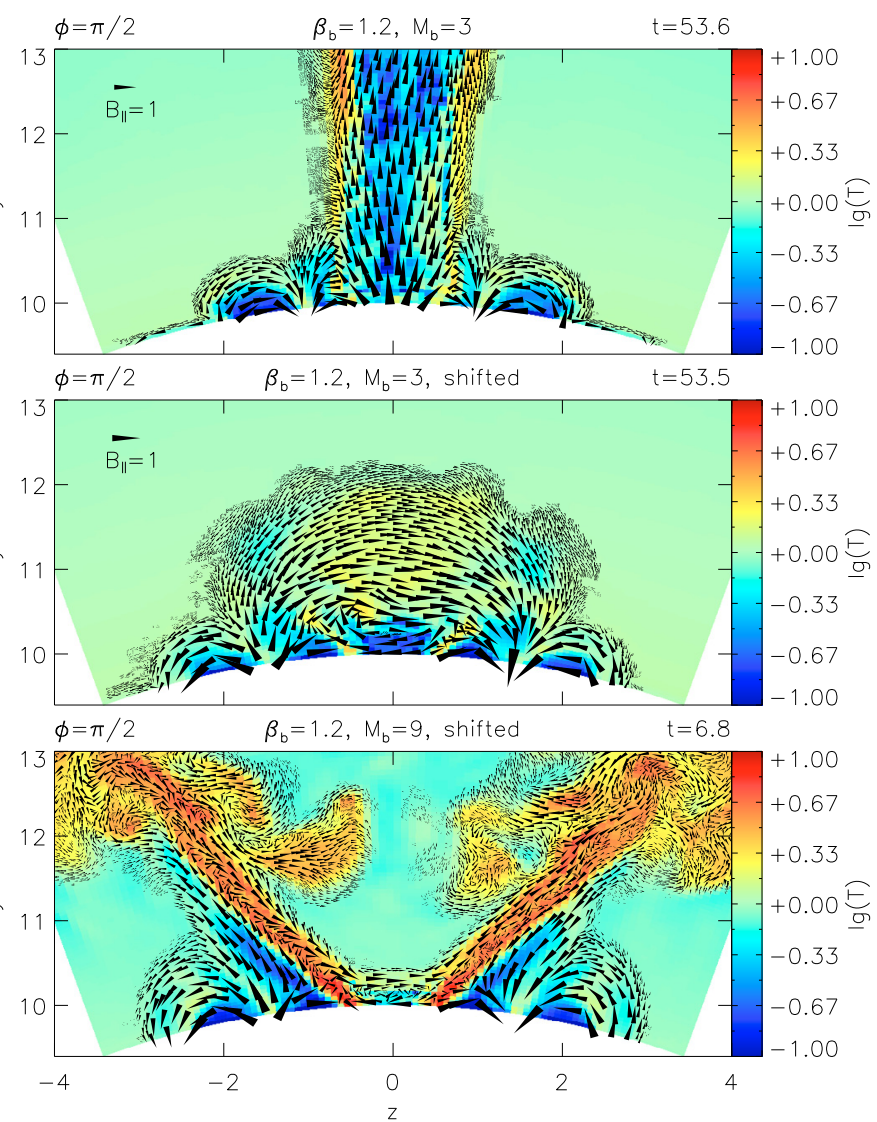

Fig. 7. Parallel magnetic field (wedges) and temperature (color) in a meridional slice through the jet in a simulation with shear (top panel) and in two simulations without shear (middle/bottom panel). Collimated, magnetically driven jets form only in cases where a toroidal field develops through a shear of the magnetic field.

comparison, close to zero at most radii and times. Accordingly, visualizations of the field lines indicate that most lines remain connected to the lower boundary at both ends until the jet crosses the upper boundary.

Figure 10 shows the average value of various quantities across the flow, where we calculate the average of $X$ as $\int X v_{r} \mathrm{~d} A / \int v_{r} \mathrm{~d} A$ over a surface for which $r=$ constant. The average velocity increases mainly below $r \approx 20$, at which point it exceeds the average fast magnetosonic speed $v_{\mathrm{fm}}^{2}=c_{\mathrm{s}}^{2}+v_{\mathrm{A}}^{2}$. The peak velocity (maximum of $v_{r}$ on $r=$ constant) is constant beyond $r \approx 30$ and is twice as large as the average velocity (4 instead of 2). The average density is at all radii significantly lower than the atmospheric value, and the temperature $\left(c_{\mathrm{s}}^{2}\right)$ is higher than the atmospheric value. The transversal (toroidal) magnetic field dominates over the radial component at medium and large distances. The magnetic pressure dominates over the gas pressure by a factor of about 2-4 at all distances in the jet.

The dependence of the energy flow rates on distance provides information about energy transformations occurring in the jet. The total energy flow rate $\mathcal{E}_{\text {tot }}(t, r)$ is obtained from a surface integral over the components of the radial energy flux

$$
\underbrace{\frac{1}{2} \rho v^{2} v_{r}}_{\text {kinetic }}+\underbrace{\frac{\gamma}{\gamma-1} p v_{r}}_{\text {thermal enthalpy }}+\underbrace{\rho \Phi v_{r}}_{\text {grav. potential }}+\underbrace{S_{r}}_{\text {magnetic enthalpy }},
$$

where

$S_{r}=\frac{1}{4 \pi}\left(B_{\vartheta}^{2} v_{r}+B_{\varphi}^{2} v_{r}-B_{\vartheta} B_{r} v_{\vartheta}-B_{\varphi} B_{r} v_{\varphi}\right)$ is the radial component of the Poynting vector. We denote the individual components of $\mathcal{E}_{\text {tot }}$ by $\mathcal{E}_{\text {kin }}, \mathcal{E}_{\text {thrm }}, \mathcal{E}_{\text {grav }}$, and $\mathcal{E}_{\text {mag }}$ in order of their appearance in Eq. (3). The components of the magnetic enthalpy flow rate are denoted by $\mathcal{E}_{\text {mag }}^{1 \ldots . .4}$ in order of their appearance in Eq. (4). The energy flow rates in the present simulation are plotted in Fig. 11. The plot shows how magnetic enthalpy is converted into kinetic and potential energy. The conversion is efficient in that less than $25 \%$ of the initial $\mathcal{E}_{\text {mag }}$ remains in the jet when it reaches the upper boundary. Thermal energy is less important, $\mathcal{E}_{\text {thrm }}$ being reduced by only half as many units as $\mathcal{E}_{\text {mag. }}$. The most important components of $\mathcal{E}_{\text {mag }}$ are $\mathcal{E}_{\text {mag }}^{2}$, which is associated with the advection of the azimuthal field and, at low radii, $\mathcal{E}_{\text {mag }}^{4}$, the work performed by the azimuthal flow against the azimuthal component of magnetic stress. The component $\mathcal{E}_{\mathrm{mag}}^{3}$ is virtually zero. In summary, the qualitative behavior of the energy flow rates is similar to those in simulations of jets generated by twisting a uniformly polarized large-scale magnetic field (Papers I and II).

The jet exhibits plenty of substructure (see Fig. 9). Above $r \approx 20$, it is affected by recurrent instabilities that divert its course away from the $y$-axis by several degrees. Stirred up ambient material contributes to the unstable behavior. Occasionally, the jet develops a pronounced helical shape, which is characteristic of kink instabilities. The helix makes approximately one complete turn within the computational volume, out of which it is rapidly advected. For comparison, the magnetic pitch is $\lesssim 10$ inside the jet (cf. Fig. 8), which is at least a factor 10 below the wavelength of the prominent instability. The largest deflections from the central axis are approximately $10^{\circ}$. Being continually recreated at its base, the jet survives in a time-averaged sense.

\subsection{Jets from a magnetic arcade on a differentially rotating surface}

We also produced jets with emerging field loops (setup E) on a differentially rotating surface (see sketch in Fig. 1c). These jets are mostly the result of thermal buoyancy, driven by dissipative heating of the near-disk atmosphere. Nonetheless, the presence of a magnetic field is required to launch a directed flow.

The simulations cover the same physical range as the one presented in the preceding section. The resolution is $400 \times 256 \times$ 256 , so that the diameter of the differentially rotating surface is resolved with about 25 cells. The longest simulation, which also produces the most efficient jet, has the parameter values $\left(\beta_{\mathrm{b}}=\right.$ $\left.1.2, \mathrm{M}_{\mathrm{b}}=15\right)$. It ran for 14 wallclock days on 96 processors and covers almost two jet crossings, the upper boundary being reached at $t \approx 62$.

In the jet of the preceding section, Poynting flux was the main source of energy for accelerating the jet. This is clearly different here. The simulation with $\left(\beta_{\mathrm{b}}=1.2, \mathrm{M}_{\mathrm{b}}=15\right)$ attains a momentary quasi-stationary state, where $\mathcal{E}_{\text {tot }} \approx$ constant in the energy flow, at $t \approx 80 \ldots 90$. In sharp contrast to Fig. $11, \mathcal{E}_{\text {thrm }}$ is the dominant component at the beginning, being a factor of $\sim 3$ greater than $\mathcal{E}_{\text {mag }}$. The equipartition point $\mathcal{E}_{\text {kin }}=\mathcal{E}_{\text {mag }}$ is very close to the lower boundary, the radius at which $\mathcal{E}_{\text {kin }}=\mathcal{E}_{\text {thrm }}$ is larger, at $r \approx 15$, and $\mathcal{E}_{\text {mag }}$ also diminishes (by about $80 \%$ ), but it is only a small fraction of the final $\mathcal{E}_{\text {kin }}$.

The excess temperature responsible for the high $\mathcal{E}_{\text {thrm }}$ increases close to the central axis next to the lower boundary and increases with the speed of rotation. The heat is apparently produced by numerical viscosity, i.e., dissipation of kinetic energy due to the coarse resolution of the rapidly rotating inner part of the disk. Unfortunately, a much higher resolution is unfeasible 

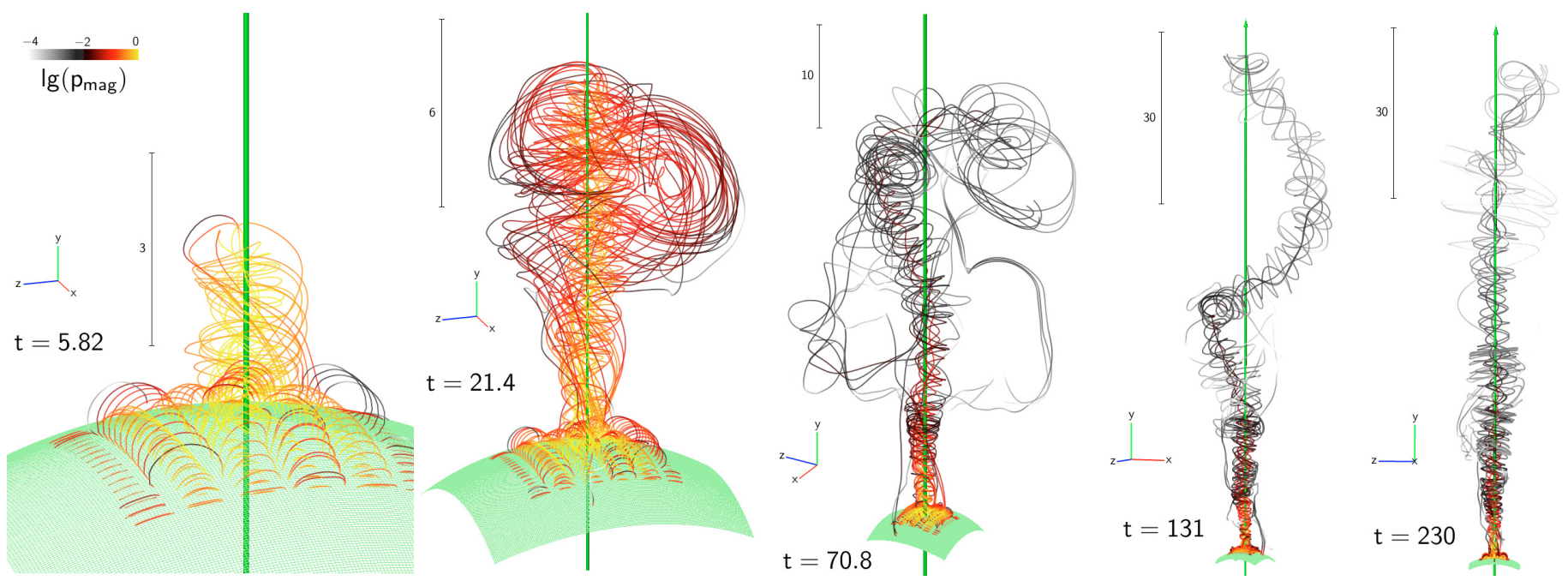

Fig. 8. Selected magnetic field lines in the simulation presented in Sect. 4.3. The color varies with the magnetic field energy. The boundary conditions create magnetic field arcades above the boundary, the arcades are twisted in the center by the imposed rotation, and a jet with helically shaped field lines forms along the axis of rotation. The jet is subject to occasional helical deformations.

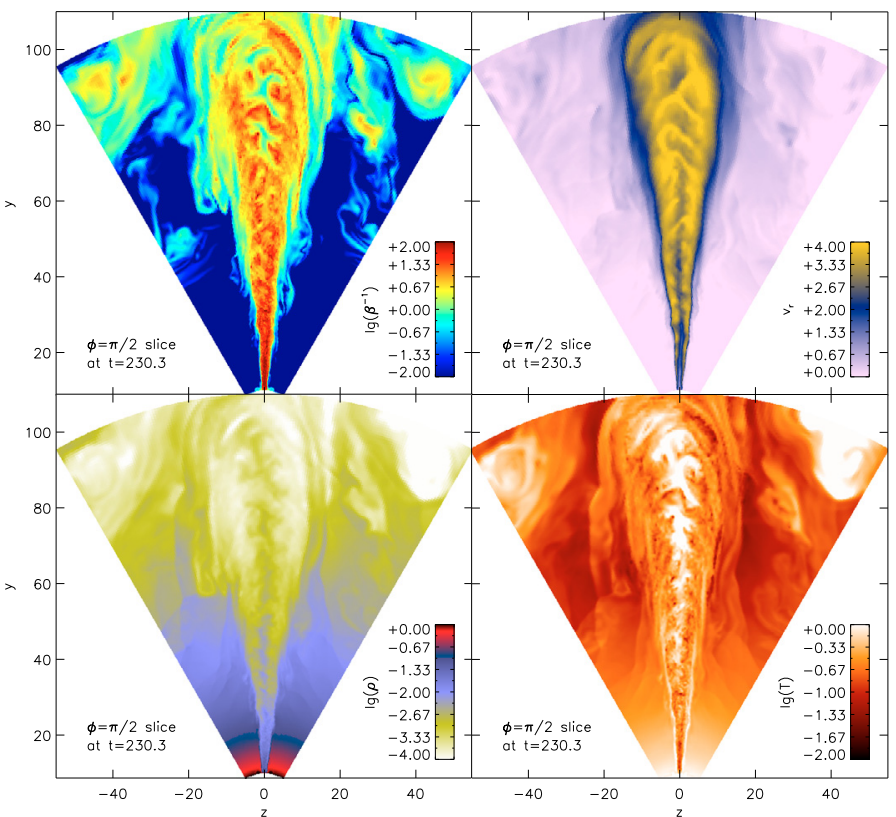

Fig. 9. Meridional slices through the jet presented in Sect. 4.3. The magnetic jet is accelerated into a non-magnetic atmosphere, penetrating through denser material. The hot, magnetic material in the environment of the upper part of the jet are remnants of previous instabilities. For movies, see the web address indicated in the footnote on page 4.

and a large $\mathrm{M}_{\mathrm{b}}$ is needed for an efficient production of $B_{\varphi}$. The simulations are therefore relevant to cases in which dissipative heating is of importance.

The appearance of the flow becomes more jetlike with increasing rotation velocity. In a simulation with $\left(\beta_{\mathrm{b}}=1.2, \mathrm{M}_{\mathrm{b}}=\right.$ $3)$, corresponding to a differential rotation velocity of about 1.1 of the outer loops' footpoints, the jet terminates at a few disk radii from the source in a tightly wound helix, similar to the free end of a garden hose. With $\mathrm{M}_{\mathrm{b}}=9$, the flow has a turbulent, "smoke-stack"-like appearance. The jet produced with $\mathrm{M}_{\mathrm{b}}=15$ is subject to non-axisymmetric perturbations, but is far more coherent.

Despite the main energy source being thermal, the results depend greatly on whether a magnetic field is present or not.
In a simulation with $\left(\beta_{\mathrm{b}}=10^{28}, \mathrm{M}_{\mathrm{b}}=15\right)$, i.e., with virtually no magnetic field, there is no unidirectional flow. Rather, material is ejected sideways as well as in the upward direction (see Fig. 12). The simulation soon crashes, presumably because too much heat accumulates. The same simulation with $\beta_{\mathrm{b}}=1.2$ is well collimated and the flow continues effortlessly through the densest parts of the atmosphere.

\section{Summary and discussion}

We have generated collimated outflows in numerical simulations by rotating the footpoints of arcade-shaped magnetic loops. The longest of these jets cross a computational domain that is two orders of magnitude larger than the size of the source.

Flow acceleration by means of dissipative as well as nondissipative processes has been observed. The latter relies on the presence of shear in the form of the differential rotation of the footpoints of individual field loops, which leads to the development of a toroidal magnetic field component. The resulting jet has a helical magnetic field structure with field lines running back to the source outside the jet.

Uniformly rotating magnetic loops have been found to be markedly less effective at producing outflows; in fact, no jetlike outflows were observed for uniformly rotating arcades within the numerically accessible parameter range. The shear between the rotating loop and the stationary atmosphere surrounding it also produces a toroidal field component, but this appears to be much less effective than direct shearing of field lines inside the rotating source.

We have found that the continuation of the outflow beyond the initial transient depends on the way that the magnetic field is maintained at the lower boundary. Differential rotation acting on a non-axisymmetric field quickly dissipates the field by convective expulsion, the result being that the jet is "choked off" at the base. We have compensated for this by adding an inflow of magnetic field at the base. This would correspond, for example, to the emergence of loops of magnetic field into the atmosphere of an accretion disk.

Apart from geometric requirements, the most important parameter for efficient jets was found to be the (differential) speed of rotation of the footpoints. In the models calculated, the 

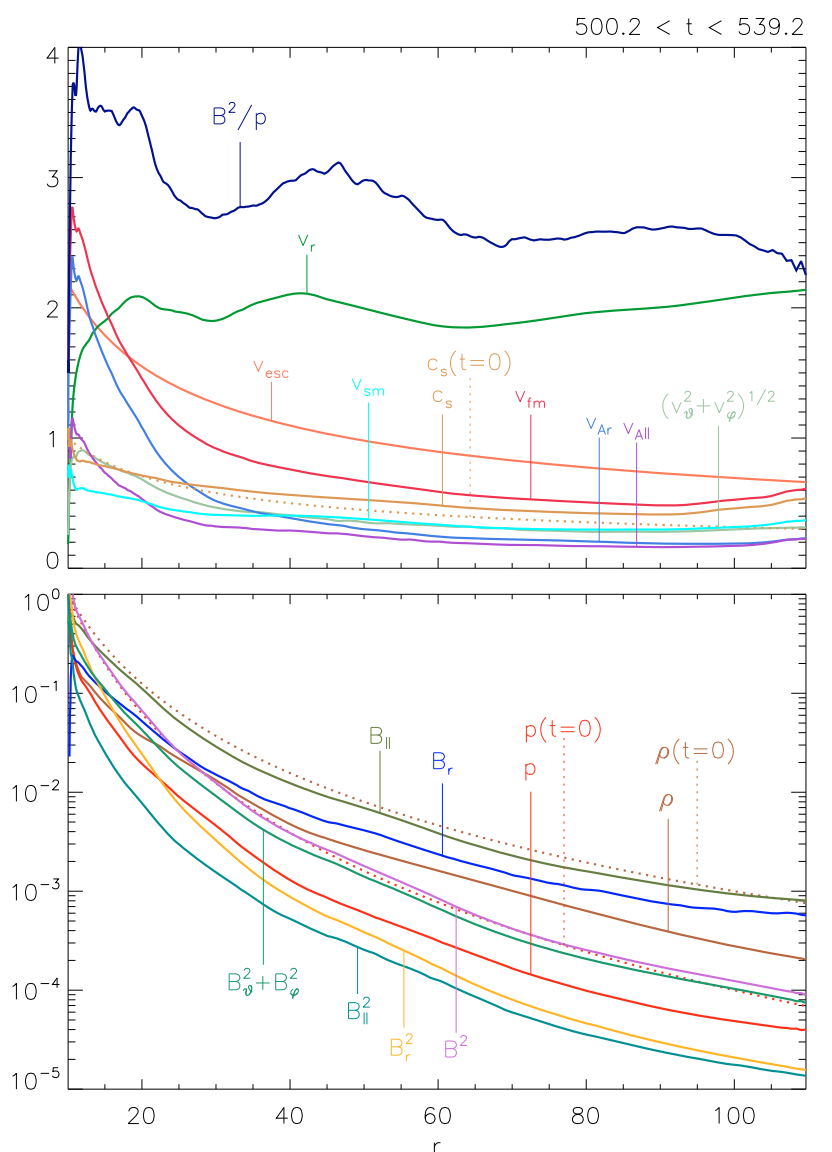

Fig. 10. Spatial and time-averaged values of various quantities in the simulation presented in Sect. 4.3. The dotted lines represent the initial atmospheric value, and the $\|$ symbol stands for "parallel to $\boldsymbol{v}$ ".

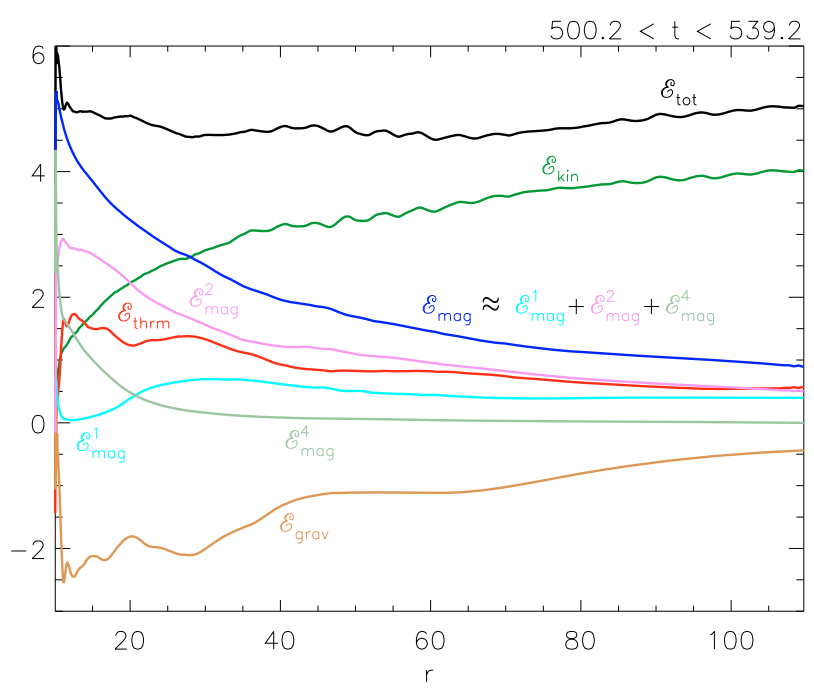

Fig. 11. Energy flow rates in the jet presented in Sect. 4.3. Magnetic enthalpy (integral over Poynting flux, blue line) is converted into kinetic energy (green line) and potential energy (brown line).

necessary speed is of the order of the escape velocity from the disk. This is more than what is needed in simulations with a long-scale poloidal initial field (e.g., those presented in Paper I). Consequently, the critical points (sonic, Alfvén, fast magnetosonic) and the equipartition point between the magnetic and kinetic energy flow rates are reached at smaller distances. The conversion of magnetic enthalpy (and the components thereof)
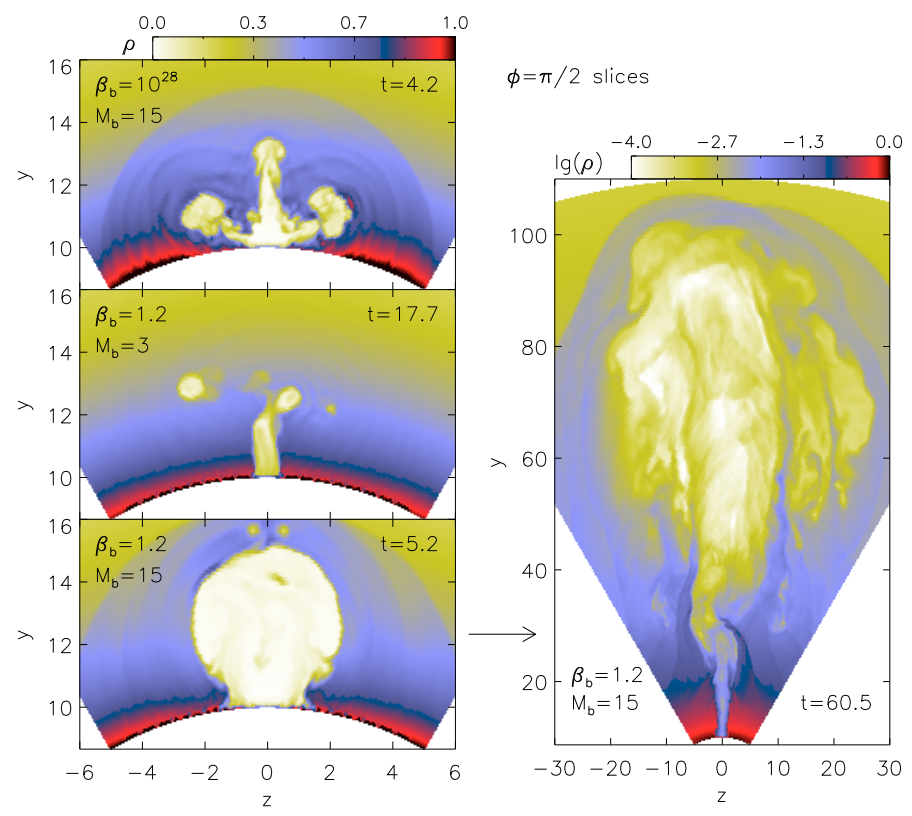

Fig. 12. Meridional slices through the jets presented in Sect. 4.4. A jet forms only if a magnetic field is present and the rotation velocity is sufficiently large. Movies of these jets can be found at the web address indicated in the footnote on page 4 .

into kinetic energy is similar apart from that, being also fairly efficient.

In the adiabatic calculations presented here, thermal energy from hydrodynamic and magnetic dissipation remains in the flow. This is in contrast to our earlier calculations (Papers I and II), where we consider optically thin environments in which much of the dissipated energy is lost by radiation. The present models assume no energy loss and are most relevant for optically thick conditions. In the stratified atmosphere of these models, heating contributes to the flow by thermal buoyancy. In some of the cases presented (Sect. 4.4), this is the dominant driving mechanism. Although a wound-up magnetic field is also present in these buoyant plume flows, they probably do not qualify as magnetically driven. The non-axisymmetric "stirring" by the rotating magnetic field at the base that drives them may, however, be relevant for the case of a (rapidly) rotating core inside a dense stellar envelope. Even though its field configuration is not of the right kind to produce a magnetically powered outflow, dissipation of rotational energy by "stirring" may still have a powerful effect on the envelope.

The magnetic field in the jets presented here is strongly wound, the field lines making many turns along the length of the jet (see Fig. 8 for an example). Current-driven instabilities, in particular kink modes, are therefore to be expected and can indeed be found in the simulations. The turbulent wiggling of the jet increases the interaction with the ambient medium, leading to an increased entrainment of material. At large distances (larger than those covered by the simulations), it is possible that the field configuration is affected by instability-induced dissipation of magnetic energy, presumably with dynamical consequences for the jet. The effect would likely be the same as in jets launched by large-scale magnetic fields (see Paper II for that). Kelvin-Helmholtz instabilities at the interface between the jet and the ambient medium are suppressed by the relatively coarse resolution used in the simulations.

The final state of the jet, at large distances from the source, depends on the impact of instabilities in the region beyond the 
computational volume of the simulations presented here. It is reasonable to believe that the jet continues as a ballistic flow when the instabilities cease to be effective (due to the expansion of the jet and a possible decay of the toroidal field). A "disruption" of the jet would require a strong interaction with the medium into which it propagates; the atmosphere at large distances is probably too thin for that.

Although the simulations presented here demonstrate possible forms of rotation-induced acceleration, they are probably not realistic models of true accretion disks. One may wonder what happens in a more realistic scenario, where more complicated or chaotically arranged magnetic field loops emerge from the disk. Obviously, magnetic reconnection would likely play a major role in such a scenario. Nonetheless, the surviving magnetic field is stretched out in the azimuthal direction by the rotation, and the free energy in the toroidal field is transformed into an outflow. Temporary fluctuations are likely to occur if the supply of magnetic field loops is not continuous. The coherent length of the jet will then be determined by the speed with which reconnection processes destroy the initial field by which the flow is produced. The jet may flare up anew when new loops emerge. This effect could be responsible for the temporary fluctuations in gamma ray bursts.

Loops of magnetic field can also be found in the solar corona, where their evolution is probably connected with explosive ejections of material (CMEs, see e.g., Forbes et al. 2006, for a review). One general idea is that the differential rotation and convective motions at the solar photosphere produce shearing, and the energy in the sheared/twisted field is set free in an explosive event (e.g., van Ballegooijen \& Martens 1989; Zuccarello et al. 2009). In contrast to the jet models discussed here, the footpoints of a solar magnetic arcade is unlikely to be twisted by (pure) azimuthal motion. Non-azimuthal shearing is unlikely to produce an extended helical field (as in Fig. 4) and the plasma acceleration is hence different from that in a magnetically driven jet. The energy in CMEs also appears to be first accumulated and then released in a sudden event, much unlike the rather smoothly rising jets presented here.
Acknowledgements. The author thanks H. C. Spruit for fruitful discussions and a careful reading of the manuscript, and M. Obergaulinger for providing his MHD code.

\section{References}

Bisnovatyi-Kogan, G. S., \& Ruzmaikin, A. A. 1976, Ap\&SS, 42, 401 Blandford, R. D. 1976, MNRAS, 176, 465

Ciardi, A., Lebedev, S. V., Frank, A., et al. 2009, ApJ, 691, L147

De Villiers, J.-P., Hawley, J. F., Krolik, J. H., \& Hirose, S. 2005, ApJ, 620, 878

Evans, C. R., \& Hawley, J. F. 1988, ApJ, 332, 659

Fendt, C. 2009, ApJ, 692, 346

Forbes, T. G., Linker, J. A., Chen, J., et al. 2006, Space Sci. Rev., 123, 251

Galeev, A. A., Rosner, R., \& Vaiana, G. S. 1979, ApJ, 229, 318

Harten, A. 1983, J. Comput. Phys., 49, 357

Hsu, S. C., \& Bellan, P. M. 2005, Physics of Plasmas, 12, 2103

Kato, Y., Mineshige, S., \& Shibata, K. 2004, ApJ, 605, 307

Krichbaum, T. P., Alef, W., Witzel, A., et al. 1998, A\&A, 329, 873

Kudoh, T., Matsumoto, R., \& Shibata, K. 2002, PASJ, 54, 267

LeBlanc, J. M., \& Wilson, J. R. 1970, ApJ, 161, 541

LeVeque, R. J. 1992, Numerical Methods for Conservation Laws, 2nd edn., ETH Zürich: Lectures in mathematics (Birkhäuser)

Lynden-Bell, D. 2003, MNRAS, 341, 1360

Moll, R. 2009, A\&A, 507, 1203

Moll, R., Spruit, H. C., \& Obergaulinger, M. 2008, A\&A, 492, 621

Obergaulinger, M. 2008, Ph.D. thesis, Max-Planck-Institut für Astrophysik, Garching bei München

Parker, E. N. 1963, ApJ, 138, 552

Romanova, M. M., Ustyugova, G. V., Koldoba, A. V., Chechetkin, V. M., \& Lovelace, R. V. E. 1998, ApJ, 500, 703

Shepherd, D. S., Claussen, M. J., \& Kurtz, S. E. 2001, Science, 292, 1513

Suresh, A., \& Huynh, H. 1997, J. Comput. Phys., 136, 83

Tadhunter, C., Marconi, A., Axon, D., et al. 2003, MNRAS, 342, 861

Toro, E. F., \& Titarev, V. A. 2006, J. Comput. Phys., 216, 403

Tout, C. A., \& Pringle, J. E. 1996, MNRAS, 281, 219

Turner, N. J., Bodenheimer, P., \& Różyczka, M. 1999, ApJ, 524, 129

Uzdensky, D. A., \& Goodman, J. 2008, ApJ, 682, 608

van Ballegooijen, A. A. 1989, in Accretion Disks and Magnetic Fields in Astrophysics, ed. G. Belvedere, 99

van Ballegooijen, A. A., \& Martens, P. C. H. 1989, ApJ, 343, 971

Zel'dovich, Y. B. 1956, JETP, 31, 154

Zuccarello, F. P., Jacobs, C., Soenen, A., et al. 2009, A\&A, 507, 441 